Binghamton University

The Open Repository @ Binghamton (The ORB)

Winter 1-15-2018

\title{
Analysis for Science Librarians of the 2017 Nobel Prize in \\ Physiology or Medicine: The Life and Work of Jeffrey C. Hall, Michael Rosbash, and Michael W. Young
}

Neyda V. Gilman

ngilman@binghamton.edu

Follow this and additional works at: https://orb.binghamton.edu/librarian_fac

Part of the Biochemistry Commons, Genetics and Genomics Commons, and the Library and Information Science Commons

\section{Recommended Citation}

Gilman, N. V. (2018). Analysis for science librarians of the 2017 Nobel Prize in Physiology or Medicine: The life and work of Jeffrey C. Hall, Michael Rosbash, and Michael W. Young. Science \& Technology Libraries, , 1-26. doi:10.1080/0194262X.2017.1412848

This Article is brought to you for free and open access by the University Libraries at The Open Repository @ Binghamton (The ORB). It has been accepted for inclusion in Library Scholarship by an authorized administrator of The Open Repository @ Binghamton (The ORB). For more information, please contact ORB@binghamton.edu. 


\title{
Analysis for Science Librarians of the 2017 Nobel Prize in Physiology or Medicine: The Life and Work of Jeffrey C. Hall, Michael Rosbash, and Michael W. Young
}

\begin{abstract}
Jeffrey C. Hall, Michael Rosbash, and Michael W. Young are the 2017 Nobel Prize in Physiology or Medicine Laureates, having earned the award for their "discoveries of molecular mechanisms controlling the circadian rhythm." They identified the genes involved in the circadian rhythm mechanism and explained how all of the different pieces of the mechanism work together. These discoveries explain how the biological cycles of Earth's organisms correspond to the rotation of the planet, acting as an inner clock. Their research also provides further opportunities to continue learning about the role of circadian rhythm and its relationship to human health (Nobelprize.org 2017a).
\end{abstract}

KEYWORDS 2017 Nobel Prize in Physiology or Medicine, Jeffrey C. Hall, Michael Rosbash, Michael W. Young, Circadian

\section{INTRODUCTION}

The term "circadian rhythm" refers to the daily cycles that almost all biological organisms go through. The word circadian comes from the Latin circa (about) and dies (day), due to the cycles taking about a day (Yeh 2002). Circadian rhythms have been evolutionary conserved throughout history, allowing organisms from bacteria to humans to adapt to life on a planet with a twenty-four hour rotation cycle (Nobelprize.org 2017b).

Knowledge of circadian rhythms has been around since the eighteenth century, but it was not until the twentieth century that scientists really began to understand both the importance and the mechanism of these cycles. The 2017 Nobel Prize in Physiology or Medicine awardees have spent their careers researching circadian rhythms. They independently identified a piece of the mechanism behind circadian 
rhythms almost thirty years ago. Since then, they have all continued to discover more of the pieces and show how they all work together. Currently, these biological rhythms are known to be important in human health, and the health of most life on the planet, but much is still unknown.

\section{THE SCIENCE}

Great science can often start from the simplest of observations. In the 1700s, the French astronomer Jacques d'Ortous de Mairan took notice of a plant's cyclical opening and closing of its leaves (Nobelprize.org 2017a). He noted that the leaves of a mimosa plant opened towards the sun during daytime, but as night came on the leaves closed. In order to determine if this action was in response to sunlight, he placed plants in a dark room and continued to monitor them. He observed that even though the plants were not exposed to any light, the leaves continued to follow the same pattern of opening during the day and closing at night. In describing these observations, the actions of the plants were compared with those of "invalids in their beds" who are able to differentiate between night and day (Ward 1971). These observations were likely the first indication of biological clocks, but Jacques d'Ortous de Mairan's simple experiment was still not sufficient to explain how the cycle was happening (Nobelprize.org 2017b). Although the plants continued following their cyclical pattern in darkness, more research would be needed to prove that circadian rhythms were driven by an internal, or endogenous, source. What exactly controls circadian rhythms was debated for over two hundred years until studies started suggesting that the mechanism is genetic.

\section{THE GENES/PROTEINS}

In the early seventies, Ronald Konopka and Seymour Benzer (1971) published research identifying the first gene associated with circadian rhythms, the period (per) gene. In their study, they isolated three Drosophila mutants that had drastically different cycles from the normal twenty-four hour cycle; one cycle became 19 hours $\left(p e r^{S}\right)$, another changed to 28 hours $\left(p e r^{L}\right)$, and the third was arrhythmic with a 
The Life and Work of Jeffrey C. Hall, Michael Rosbash, and Michael W. Young

complete lack of rhythm $\left(p e r^{0}\right)$. Konopka and Benzer expressed curiosity at the fact that these first three clock mutations had such varying phenotypes and hypothesized that other clock genes and mutations were likely to be discovered. They also noted that the effects of the mutations were observed on both the pupal and adult cycles, suggesting that the biological clock persists through metamorphosis. A later study by Michael W. Young indicated that not only does the clock itself persist, so does the mechanism that controls it (Sehgal, Price, and Young 1992). Additional studies on per showed that it does more than affect circadian rhythms; it was shown to also affect the fluctuations of male Drosophila courtship songs (Kyriacou and Hall 1980).

Although the first gene had been discovered, it was assumed there were more to be identified. It was also unknown how this gene, and the others that were thought to exist, actually affected circadian rhythm. Thirteen years after Konopka's and Benzer's study, two studies isolating per were published. In December of 1984, Michael W. Young, working with colleagues Bargiello and Jackson, published the results of their work restoring circadian rhythm to Drosophila by period gene transfer. They were able to restore circadian rhythm to an arrhythmic fly by introducing a per gene segment using a method called $\mathrm{P}$ element-mediated transformation, which is a Drosophila specific DNA transferring technique (Bargiello, Jackson, and Young 1984). At the same time that Young was working on this research, Michael Rosbash and Jeffrey C. Hall were doing similar work at their institution. It was also in December of 1984 that Rosbash and Hall, in collaboration with their colleagues Zehring, Wheeler, Reddy, Konopka, and Kyriacou, published the results of their use of per P element-mediated transformation (Zehring et al. 1984).

P elements refer to Drosophila-specific sections of DNA that move around within the genome and have been found to be incredibly useful when it comes to the manipulation of genes (Cooley, Kelley, and Spradling 1988). These sections of DNA are called transposons, or transposable elements, and they are found in almost all organisms (Pray 2008). Transformation refers to DNA that is transferred into a 
The Life and Work of Jeffrey C. Hall, Michael Rosbash, and Michael W. Young

chromosome it did not come from. Thus, P element-mediated transformation is a laboratory technique that uses P elements to insert mutations into Drosophila genes. These new mutations, along with nearby segments of DNA, can be cloned immediately, allowing the DNA to be mapped and wild-type DNA recovered (Sehgal et al. 1991).

In 1988, Rosbash, Hall, and colleagues discovered the per gene protein PER (Siwicki et al. 1988). The size of this protein is affected by the mutations Konopka and Benzer discovered with the $p e r^{0}$ mutation. This mutation creates a stop signal causing only part of the protein to be produced, leading to the arrhythmic cycle observed (Sehgal et al. 1991). Rosbash's and Hall's research that identified PER also found that the protein's levels oscillated throughout the day and night in a twenty-four hour cycle. This observation was made by generating antibodies to specific PER peptides, or sections of the protein made up of short chains of amino acids. The antibodies were bound to the PER peptides which were then viewed after immunoprecipitation made them available to be ran on Western Blot assays (Siwicki et al. 1988). Immunoprecipitation is a process where antibodies are bound to proteins and then the antibody-protein complex is precipitated out of solution by coupling the antibody to a substrate. Western Blot refers to a technique that separates proteins on a gel according to size and targets specific proteins to be viewed by binding them with antibodies (Mahmood and Yang 2012). Rosbash and Hall looked for PER in photoreceptors, neuronal cell bodies, and small cells from specific central nervous system locations. There were consistent differences between the night and day levels of PER, showing the circadian pattern. Significantly, this pattern was still observed when the flies were placed in constant darkness. This finding is significant because it suggests that the circadian rhythm mechanism is not ran by external factors such as light, but rather is an endogenous system. Light acts to set a circadian cycle, but is not necessary to maintain it. Changes to the twenty-four hour cycle can occur both through the three per point mutations initially shown in Konopka's and Benzer's 1971 work, as well as through an increase or decrease in PER levels (Sehgal et al. 1991). 
The Life and Work of Jeffrey C. Hall, Michael Rosbash, and Michael W. Young

Hall and Rosbash continued working with per and a couple of years after their PER discovery they published new findings of a feedback loop between PER and per messenger RNA (mRNA) (Hardin, Hall, and Rosbash 1990). Since the head of Drosophila had been shown to be the location of the "circadian oscillator" (Konopka, Wells, and Lee 1983) Hall, Roshbash, and Hardin took per mRNA from Drosophila heads to examine the change in levels throughout the day and night. They looked at mRNA and PER levels from wild type flies and flies with per mutations. The results suggested that not only do the levels of mRNA affect the levels of PER, the levels of protein also affect the levels of mRNA. The cycle Hall and Rosbash hypothesized is that per mRNA is transcribed from the per gene in the nucleus and then transported into the cytoplasm where the protein, PER, is created. PER then somehow travels into the nucleus where it blocks the transcription of more mRNA. The blocking of mRNA transcription leads to an inability to make PER, which in turn decreases the amount of PER available to continue blocking mRNA transcription, starting the cycle anew (Hardin, Hall, and Rosbash 1990). The researchers understood that PER regulates itself in this cyclical rhythm. The rhythm was observed, but the mechanism was yet unknown.

Meanwhile, Young was independently searching for more circadian related genes by using P elementmediated transformation. He did this by looking at eclosion and locomotor activity of flies that had a mutation inserted by $\mathrm{P}$ element. Eclosion, the act of emerging from a pupal state, and locomotor activity are the two characteristics that Konopka and Benzer initially looked at, and that have continued to be used to observe chances in circadian biology. Some of the mutations he found that seemed to be related to circadian rhythm are glass, $m 120$, and aj42 (Sehgal et al. 1991). The andante (and) and clock (clk) mutations had previously been discovered and shown to affect circadian rhythms (Konopka 1986). And lengthens cycles by about two hours while clk shortens cycles by about an hour and a half. Around the time Young was identifying these genes, Dushay, Roshbash, and Hall (1989) showed that the 
The Life and Work of Jeffrey C. Hall, Michael Rosbash, and Michael W. Young

disconnected gene, a gene known to play a role in neuronal cell recognition, is another circadian rhythm gene.

In 1994, Young discovered another gene and published back-to-back articles on it in an issue of Science.

This gene, called timeless (tim), causes arrhythmic eclosion and locomotor activity in Drosophila and was shown to suppress per mRNA rhythms (Sehgal et al. 1994). In addition to affecting the oscillation of per mRNA, tim also affects PER (Vosshall et al. 1994). Rosbash and Hall had already discovered the per mRNA-PER inhibitory feedback loop, and PER had been observed in the nucleus of various Drosophila cells in levels that oscillated with a circadian rhythm, but it was still unknown how the protein produced in the cytoplasm was actually getting into the nucleus (Edery et al. 1994, Hardin, Hall, and Rosbash 1990). Young's tim research began to shed light on this problem with the Vosshall article which describes how the tim mutation results in no nuclear staining of PER. Although the method was still not fully understood, their research showed that the tim protein (TIM) binds to PER in order to enter the nucleus, and also suggested that PER is unstable in the cytoplasm; thus without TIM, PER would not be able to enter nuclei and would degrade quickly.

Young continued to build on his research showing that the binding of PER and TIM is what controls the timing of PER entering the nuclei, which in turn affects the length of the circadian cycle (Gekakis et al. 1995). The section of PER that TIM binds to, referred to as the PAS domain, is thought to be the same section that contains the signal for PER to stay in the cytoplasm (Vosshall et al. 1994). Young and others also discovered that although light has no effect on per or PER, the TIM protein degrades rapidly in light (Myers et al. 1996). This means that TIM and the TIM-PER compound accumulate in the cell overnight when it is dark, but TIM is degraded with the morning light. Since PER is unstable when not bound to TIM, it too starts to degrade. It has also been observed that both proteins TIM and PER provide a feedback loop to both genes tim and per. The gene double-time ( $d b t)$, also discovered by Young, was shown to affect PER stability, adding another component to the circadian machinery (Price et al. 1998). 
The Life and Work of Jeffrey C. Hall, Michael Rosbash, and Michael W. Young

The $d b t$ protein DBT, a homolog to the mammalian enzyme casein kinase 1 , affects PER's stability when necessary to regulate the circadian twenty-four hour cycle (Rothenfluh, Abodeely, and Young 2000, Nobelprize.org 2017b, Kloss et al. 1998). See Figure 1 for a simplified representation of the cycle. All of this research taken together shows the mechanism behind circadian rhythms (Myers et al. 1997, Zeng et al. 1996) and is the reason these three scientists received the 2017 Nobel in Physiology or Medicine.

[Figure 1. Circadian Mechanism]

FIGURE 1 A simplified illustration of the genetic circadian mechanism.

Around the time that Young was discovering double-time, Hall and Rosbash discovered two other critical circadian genes: $d C l o c k(C l k)$ and cycle $(c y c)$. The articles discussing the discovery and research into these genes were published back-to-back in a 1998 issue of Cell. The $d$ Clock gene was identified as being homologous to an already identified mouse clock gene, hence the $d$ for Drosophila. This finding in itself is significant since it supports the conservation of these genes throughout evolution. Both Clk and cyc were shown to affect circadian rhythm by affecting per and tim. Hall, Rosbash, and their team speculated that Clk and cyl produce proteins (CLK and CYC) that bind together and then the CLK-CYC compound binds to both genes per and tim, affecting their transcription (Allada et al. 1998, Rutila et al. 1998).

Around the same time that they identified Clk and cyc Rosbash, Hall, and colleagues identified cry. Cry is a cryptochrome gene that codes for a photoreceptor that resets circadian rhythms. It does this by producing a protein, CRY, which binds to TIM when exposed to light (Emery et al. 1998). Once CRY binds to TIM, TIM begins to degrade, meaning it cannot bind to PER and transport it into the nucleus. This causes the system to pause until dark when TIM can accumulate uninhibited by CRY and resume binding 
The Life and Work of Jeffrey C. Hall, Michael Rosbash, and Michael W. Young

to PER (Rockefeller Univesrity 2017). The identification of this gene provided understanding of the mechanism by which the biological clock can be set and synchronized by light.

Cry, period, timeless, double-time, Clock, and cycle are parts of a small group of genes that play a role in the circadian clock of Drosophila. These genes affect the length of biological, behavioral, or physiological rhythms. The other genes in this group are shaggy, PAR domain protein 1, vrille, and casein kinase 2. Young's lab has continued with the work of circadian genes, and in the process has created microarrays of all 14,000 Drosophila genes (Rockefeller Univesrity 2017). Since the first genes were discovered, the field of chronobiology, or circadian biology, has advanced. As mentioned before, the head was initially considered the location of circadian controlling cells; it has since been determined that the brain is the center for these cells but circadian genes exist in various other cells throughout the body, for most multicellular organisms (Wedell 2017, Plautz et al. 1997). The identification and further understanding of all of these genes adds to a growing insight of how the clock mechanism works, influences itself, and remains stable.

\section{EFFECTS/IMPORTANCE}

It is now known that circadian rhythms are autonomous oscillators that biological organisms need in order to thrive on Earth. Hall, Rosbash, and Young identified the mechanism that controls these rhythms. Their work opened up and continues to expand the broad field of chronobiology including the sub-discipline of chrono-medicine, which is thriving (Roenneberg and Merrow 2016). Current research continues to explore the correlations of circadian rhythm with numerous diseases and the implications for human health and lifestyle, with many questions still unanswered. The 2017 laureates not only helped spawn these questions, but also provided themselves and others with the tools and knowledge to help answer them (Wedell 2017).

Biological clocks are tied to many parts of human physiology including aspects of health, lifestyle, and diseases (Nobelprize.org 2017b). In a recent New York Times article, Dr. Frank A.J.L. Scheer, Associate 8 I P a g e 
Professor of Medicine at Harvard Medical School and Director of the Medical Chronobiology Program at Brigham and Women's Hospital, stated that circadian rhythm is "intimately linked to our health and disease, including diabetes, obesity, cancer and cardiovascular disease" (Kolata 2017). Circadian genes have even been identified as being at least partially responsible for determining if someone will be a "morning person" or not (Hu et al. 2016).

Some of the numerous physiological activities affected by circadian rhythm include sleep, feeding times, and hormones (Ibáñez 2017). Sleep is a common thread tied to circadian rhythm and frequent examples of how circadian rhythms impact human health include jet lag and shift work. With most cells in the body having biological clocks, activities such as extended shift work can cause a chronic misalignment of those clocks and lead to an increased risk of diseases, including cancer (Khalyfa et al. 2017, Schernhammer et al. 2001). Travelling across time zones leads to internal clocks being temporarily misaligned from external clocks, both societal and environmental. While this may not lead to increased risk of disease, it causes issues with sleeping and leads to exhaustion and feeling ill. During the announcement of the 2017 Nobel Prize in Physiology or Medicine, an attendee asked if an organism could adapt to shorter cycles than 24-hours (Nobelprize.org 2017b). In response, Professor Thomas Perlmann, Secretary of the Nobel Committee for Physiology or Medicine, noted that some humans do have altered cycles and that these alterations do result in sleep problems. The link between circadian and sleep has long been researched. When Hall, Rosbash, and Young were still actively identifying and studying major circadian genes, a study in Japan showed that the use of melatonin and methyl $\mathrm{B}_{12}$ could be successful in normalizing the circadian rhythm of those who suffer from biological rhythm disturbances (Tomoda et al. 1994). Research based on the mechanics of circadian rhythm has also led to an increase in the awareness of proper sleep hygiene (Irish et al. 2015). Future research may lead to additional treatments including pharmaceuticals targeted at specific genes (Rockefeller University 2017). 
Young's current research continues to be applicable to disorders affecting sleep as well as mood, visual functions, locomotion, metabolism, immunity, learning, and memory (Rockefeller Univesrity 2017). For example, Young discovered that when casein kinase 1 (DPT in Drosophila) does not interact properly with PER, humans develop heritable sleep disorders (Young and Kay 2001). More recent research has identified a "night owl" gene that makes it difficult for people to stay on a twenty-four hour schedule, leading to another heritable disorder, a form of delayed sleep phase disorder (DSPD) (Patke et al. 2017). Other researchers build upon the existing circadian rhythm and sleep research by using nonconventional methods more applicable to the twenty-first century. Walch, Cochran, and Forger (2016) published the results of their work that made use of a phone app, which assists traveling users by alerting them to seek dark or light environments in order to reset their internal clock and avoid jetlag. While doing this, the app also collects sleep data that the scientists then use to further their research. Recent research has also been done building on Rosbash's and Hall's research into how light effects circadian rhythm. It has been shown that there are separate sets of neurons that are divided by their sensitivity to light (Brandeis University 2017b). One set is sensitive to an increase in light, such as at dawn, while another is sensitive to light decreasing, such as at dusk. Another article discussed findings that exposure to light throughout the day actually inhibits arousal during the night (Shang et al. 2011). Additional studies have shown circadian and sleep disturbing effects after exposure to artificial light from electronic devices (Cajochen et al. 2011, Green et al. 2017).

Chronobiological research obviously looks closely at sleep, but the field covers a broad range of research. A recent Lancet article discusses how a circadian gene affects the heart muscles ability to tolerate cycling levels of oxygen (Montaigne et al. 2017). Their research suggests that due to the body's clock, heart muscles may be better able to withstand the stress of surgery, specifically aortic valve replacement, in the afternoon rather than the morning. Another recent article discusses the importance of circadian rhythm, and specifically the Clock gene, to cardiovascular health (Alibhai et al. 2017). There 
The Life and Work of Jeffrey C. Hall, Michael Rosbash, and Michael W. Young

has also been recent research into resetting or altering circadian rhythms by dietary guidelines such as intermittent fasting. These studies show benefits to health, weight, aging, and longevity (Froy 2017, Li et al. 2017).

David Baltimore, 1975 Physiology or Medicine Nobel Laureate and Caltech Professor of Biology, recognized this year's Physiology or Medicine Nobel Laureates as deserving of the prize and for providing "the basis for all following work in the critical field of chronobiology" (Kolata 2017). The laureates' work explained how cells keep time, and laid the groundwork for future research into biological rhythms and their effects on disease and health. The Howard Hughes Medical Institute's Biointeractive site has a series of lectures on circadian clocks. These lectures are given by Nobel Laureate Dr. Michael Rosbash along with Dr. Joseph Takahashi (Howard Hughes Medical Institute). They are a good resource for anyone wanting to know more about the research behind this year's Nobel award and how biological clocks work.

\section{THE “LITTLE FLIES”}

The Nobel Prize in Physiology or Medicine 2017 press release states that the winners used fruit flies as their model organism (Nobelprize.org 2017a). During Dr. Hall's interview, he noted that he felt the "little fly" should be the fourth recipient of the Nobel (Hall 2017). The fly, Drosophila, played a critical role in the research leading to this award and it continues to be vital in current research. Due to its importance and the affinity many researchers seem to feel for it, the following short section will be dedicated to the fruit fly.

An October 20th, 2017 search for "drosophila" in the indexing database Web of Science (WoS) found 148,554 results dating back to 1905 . The WoS research areas of these results included obvious fields such as biochemistry molecular biology and genetics heredity, but also biophysics, behavioral sciences, and toxicology. For the years 1905 through 1909, there is only one Drosophila-related publication 
The Life and Work of Jeffrey C. Hall, Michael Rosbash, and Michael W. Young

indexed annually in WoS, and two in 1910. After 1910, these numbers rise, jumping to eight in 1911, twenty-three by 1920, and over 6000 by 2012 (see Figure 2). One of these articles, T.H. Morgan's (1910) study on sex-inheritance in Drosophila, can be said to have kick-started the use of Drosophila in the lab. Rosbash refers to this study in at least two separate interviews. The first is during an interview for the Howard Hughes Medical Institute Biointeractive series Clockwork Genes: Discoveries in Biological Time (Howard Hughes Medical Institute). He also states it by name during the Brandeis press conference for the 2017 Nobel award (Brandeis University 2017c). As of October 23, 2017, this study was cited 253 times in WoS. However, the most highly cited Drosophila-related article in WoS, as of this date, is D.P. Bartel's 2009 article “MicroRNAs: Target Recognition and Regulatory Functions" which had been cited over 8,400 times.

[Figure 2. Drosophila Publicatiosn]

FIGURE 2 Number of Drosophila Related Publications by Year. Data source: Web of Science. Retrieved 20 October 2017.

Morgan's work with fruit flies not only led to a boon in research using Drosophila, it also led to him receiving the 1933 Physiology or Medicine Nobel for his discoveries involving the role of chromosome in heredity (Nobelprize.org). Three other Physiology or Medicine Nobel prizes were awarded in part due to Drosophila: Herman J. Muller in 1946 for explaining how genes can change; Edward B. Lewis, Christiane Nüsslein-Volhard and Eric F. Wieschaus in 1995 for their work on genes and embryonic development; and Bruce A. Beutler and Jules A. Hoffmann whose work on innate immunity earned half of the 2011 prize (Nobelprize.org 2011, 1995, Nobelprize.org).

In the mid-sixties, Aubrey Manning (1965) wrote a review on the evolution of behavior which shows the important role Drosophila played up until that point. Manning's review states that Drosophila is an 
The Life and Work of Jeffrey C. Hall, Michael Rosbash, and Michael W. Young

"excellent subject" and highlights numerous studies that used Drosophila dating back to a 1915 publication (Sturtevant 1915). Benzer's 1967 article states that Drosophila is useful in cellular function studies and that it is just as useful for behavioral mutation studies.

A 2013 look at Drosophila's use in research illustrates the organism's importance in the lab. This review discusses how Drosophila, usually Drosophila melanogaster, has been used to study sex-linked inheritance, ionizing radiation and mutations, various neuroscience and neurodevelopment questions, and of course circadian rhythms. According to the review, the early 1900s saw a desire for a new laboratory model organism. Drosophila began to fill this role when William Ernest Castle first used it in 1901 for his zoology research at Harvard University. The fly's complex nervous system and rapid reproduction made it a success in the lab and it has been a model organism ever since. In addition to its short generation times and robust nervous system, other advantages include its large numbers and the large amount of information known on the organism (Stephenson and Metcalfe 2013).

\section{THE SCIENTISTS}

\section{JEFFREY C. HALL}

\section{LIFE, EDUCATION, AND CAREER}

Jeffrey C. Hall was born in 1945 in Brooklyn, NY, USA, but grew up just outside of Washington D.C. where his father was an Associated Press reporter covering the Senate. In 1963, Hall began attending Amherst College in Amherst, MA where he became interested in genetics and was influenced by his mentor Philip Ives. By 1967, Hall was beginning his graduate studies at the University of Washington (UW) in Seattle, WA. Hall stayed at UW to pursue his PhD during which time he was introduced to Seymour Benzer. Hall earned his doctoral degree in 1971 and then went on to a position as a postdoctoral fellow in Benzer's lab at the California Institute of Technology (Caltech) in Pasadena, CA. In 1974, Hall became an Assistant Professor of Biology at Brandeis University in Waltham, MA. Hall stayed at Brandeis working with 
The Life and Work of Jeffrey C. Hall, Michael Rosbash, and Michael W. Young

Michael Rosbash for almost thirty years (Nuzzo 2005). In 2002, he retired from Brandeis and began working with Drosophila at the University of Maine (UM) (Kolata 2017). From 2005 to 2012 Hall was an adjunct faculty member at the UM's School of Biology and Ecology. He was also a UM Libra Professor for the 2008-2009 school year, teaching courses in genetics and neurogenetics (University of Maine 2017). Hall is now a Brandeis Professor Emeritus of Biology where he is known for having a "larger than life presence" and as someone who lives "narrowly on the line of social convention" (Brandeis University 2017c).

Hall has been the recipient of many awards and honors over the years. He received a National Institutes of Health Fellowship Training Grant from 1967 to 1971, and National Science Foundation and NIH Postdoctoral Fellowships from 1971 to 1973 . He was the editor of the journal Journal of Neurogenetics from 1992 to 2006 and the book series Advances in Genetics from 1995 to 2006 . He was elected to the American Academy of Arts and Sciences in 2001 and the National Academy of Sciences in 2003. He has also received such notable awards as the National Institutes of Health Research Career Development Award (1977 - 1982); Achoff's Rule Award in Chronobiology, Society for Biological Rhythms and Gordon Conference for Biological Rhythms (1992); and the McKnight Technical Innovations in Neuroscience Award (1999 - 2001) (Brandeis University). Hall also received four awards, in addition to this year's Nobel, that he shares with Michael Rosbash and Michael Young. These are the 2009 Gruber Prize in Neuroscience, the 2011 Louisa Gross Horwitz Prize for Biology or Biochemistry, the 2013 Shaw Prize in Life Science and Medicine, and the 2013 Wiley Prize in Biomedical Sciences (Columbia University , Gruber Foundation 2009a, Shaw Prize Foundation 2013c, Wiley 2013).

Throughout his career, Hall has worked with Drosophila and expressed an affection for the fly from the very beginning (Nuzzo 2005). His work involved genetic studies of behavior, looking at genes' effect on courtship as well as circadian rhythm behaviors (Brandeis University 2017a). During his Nobel interview, Hall stated that working on circadian rhythm was about half of his life's work. He mentioned that he and 
The Life and Work of Jeffrey C. Hall, Michael Rosbash, and Michael W. Young

Rosbash worked closely together and that they had many similar interests making them personally and professionally close. He also used this interview as an opportunity to express his admiration for fruit flies, stating that the organism may seem irrelevant but it has a broader significance and should be considered a major contributor (Hall 2017).

After retiring from Brandeis, Hall became interested in the American Civil War, specifically the Battle of Gettysburg. He became so knowledgeable on the topic that he taught a Brandeis course on it, provided guest lectures, and wrote a 2003 book on the topic, The Stand of the U.S. Army at Gettysburg (Nuzzo 2005). Hall continues to live in Orono, ME, or as he likes to call it, "the middle of nowhere" (Hall 2017).

\section{BIBLIOMETRICS}

As of November 11, 2017, Hall had 206 articles indexed in Web of Science (WoS). He has published steadily over his career; many years publishing over ten articles annually (see Figure 3). His most recent publication was a memorandum of his mentor and colleague, Ronald J. Konopka (Hall 2015). He has been cited over 19,000 times with an average of almost ninety-four citations per publication. Since 1994 he has had over 400 citations a year, often over 600 reaching a max of almost 1100 in 2002 (see Figure 4). His WoS h-index is eighty-one.

[Figure 3. Hall Publications]

FIGURE 3 Jeffrey C. Hall, Number of Published Items in Each Year. Data source: Web of Science. Retrieved 11 November 2017.

[Figure 4. Hall Citations]

FIGURE 4 Jeffrey C. Hall, Number of Times Cited in Each Year. Data source: Web of Science. Retrieved 11 November 2017. 
Of Hall's 206 publications, sixty-seven of them have over 100 citations each. The top five cited articles all have almost 500 or more citations (see Table 1). Hall's most highly cited articles is his 1990, Feedback of The Drosophila Period Gene Product on Circadian Cycling of its Messenger RNA Levels with 632 citations as of November 11, 2017. Not surprisingly, almost all of the journals in which Hall has published are biology-related journals, and the three he published in the most are all genetics focused (see Table 2). The journal Genetics is his most frequently published in journal.

Table 1. Top Five Most Highly Cited Papers by Jeffrey C. Hall.

\section{[TABLE 1]}

Table 2. Top Five Journals Published in by Jeffrey C. Hall.

\section{[TABLE 2]}

\section{MICHAEL ROSBASH}

\section{LIFE, EDUCATION, AND CAREER}

Michael Rosbash was born in 1944 in Kansas City, MO, USA eventually moving to Newton, MA as a child. His parents had immigrated to the USA from Nazi Germany and his father died when he was only ten and his brother was six. Has father's death made his already difficult childhood even harder and he took advantage of college to get as far away as home as possible (Shaw Prize Foundation 2013a). He attend the California Institute of Technology (Caltech) in Pasadena, CA earning a BS in Chemistry in 1965. After Caltech, Rosbash moved on to the Massachusetts Institute of Technology (MIT) to earn his doctoral degree in biophysics in 1970. Following MIT, Rosbash spent three years in Edinburgh Scotland as a postdoctoral fellow at the University of Edinburgh (Gruber Foundation 2009a). After returning to the USA, he became a faculty member at Brandeis University in Waltham, MA where he remains today. Rosbash arrived at Brandeis six months after Hall did. The two almost immediately became friends, but 
The Life and Work of Jeffrey C. Hall, Michael Rosbash, and Michael W. Young

they did not start collaborating on work until 1982 when the science began to show an overlap between Rosbash's biochemistry focus and Hall's genetics (Shaw Prize Foundation 2013a). Rosbash is currently a Brandeis Professor of Biology, a Peter Gruber Endowed Chair in Neuroscience, and a Howard Hughes Medical Institute Investigator (Brandeis University). According to nobelprize.org, Rosbash received the first Nobel awarded to a Brandeis University Researcher (Nobelprize.org). (The University of Maine is the institution associated with Hall on nobelprize.org. However, the work for which he received the award was done at Brandeis.)

During his interview with nobelprize.org, Rosbash stated that he sees Hall, Young, and himself as pioneers in linking genes to behavior. He also noted that he and Hall worked well together because they have complementary skill sets (Rosbash 2017). On his personal Brandeis webpage, Rosbash writes in depth about his lab's work stating that they are still researching circadian biology with three specific goals in mind. These goals are to gain more understanding of the timing mechanisms, to understand "how circadian gene expression regulation takes place," and to understand more about the neurons and neural circuits involved. He states that his lab also continues to work on non-circadian gene expression work (Brandeis University 2017b).

Rosbash's colleagues describe him as imaginative, creative, and curious with a desire to solve real world challenges and someone who likes interactive and rigorous work. It was noted that he is engaged on campus, has a great sense of humor, and is full of energy. He was also described as a generous person to his colleagues, fellow scientists, and his students. This generosity came out during his speech at the Brandeis Press Conference where he said he has been lucky in having "remarkable students" who made it all possible. He also thanked both the National Institutes of Health and Brandeis University, stating that he owes his career to them (Brandeis University 2017c).

Similar to Hall, Rosbash has received numerous awards and honors. He became a member of the American Academy of Arts and Sciences in 1997 and the National Academy of Sciences in 2003. He was a 
The Life and Work of Jeffrey C. Hall, Michael Rosbash, and Michael W. Young

Fulbright Fellow from 1965 to 1966, Helen Hay Whitney Fellow from 1971 to 1974, Guggenheim Fellow from 1989 to 1990, and American Association for the Advancement of Science Fellow in 2007. He became a Howard Hughes Medical Institute Investigator in 1989. Some awards he has received include the NIH Research Career Development Award (1976 - 1980) and the Caltech Distinguished Alumni Award (2001) (Brandeis University). There are also four he shares with his fellow 2017 Physiology or Medicine Laureates: the Gruber Prize in Neuroscience (2009), the Louisa Gross Horwitz Prize for Biology or Biochemistry (2011), Shaw Prize in Life Science and Medicine (2013), and the Wiley Prize in Biomedical Sciences (2013).

Rosbash is married to his wife Nadja, and has a daughter Tanya and stepdaughter Paula (Shaw Prize Foundation 2013a).

\section{BIBLIOMETRICS}

As Michael Rosbash and Jeffrey Hall had worked together for so long, they also frequently published together. However, Rosbash has published more frequently with 322 articles indexed in WoS as of November 11, 2017. His first two articles were published in 1970 and he has published annually ever since. Since 1979, Rosbash has published at least three articles a year, often publishing ten or more times a year (see Figure 5). His articles are often highly cited leading to him being cited hundreds or even a thousand times a year (see Figure 6). Rosbash has a WoS h-index of 100.

[Figure 5 Rosbash Publications]

FIGURE 5 Michael Rosbash, Number of Published Items in Each Year. Data source: Web of Science. Retrieved 11 November 2017.

[Figure 6 Rosbash Citations] 
FIGURE 6 Michael Rosbash, Number of Times Cited in Each Year. Data source: Web of Science. Retrieved 11 November 2017.

His 322 articles have 29,307 citations, with an average of ninety-one citations per article. The top five cited articles all have over 500 citations each and the top three have over 600 (see Table 3). Unlike many researchers' early publications, both of Rosbash's first publications have been cited a number of times. This is especially true for his article Messenger and Heterogeneous Nuclear RNA in Hela Cells Differential Inhibition by Cordycepin, which has been cited over 300 times. This article was published in the Proceedings of the National Academy of Sciences of the United States of America, which is Rosbash's second most frequently published in journal. The journals he has published in the most is Cell (see Table 4).

Table 3. Top Five Most Highly Cited Papers by Michael Rosbash.

[Table 3]

Table 4 .Top Five Journals Published in by Michael Rosbash.

\section{[Table 4.]}

\section{MICHAEL W. YOUNG}

\section{LIFE, EDUCATION, AND CAREER}

Michael W. Young was born in 1949 in Miami, FL, USA just a couple years before his sister. His father managed regional aluminum ingot sales and his mother was a legal secretary (Shaw Prize Foundation 2013b). Young's parents encouraged his interest in science from a young age with many science related gifts. One of these gifts was a book on biology, which happened to explain what was happening to a plant he had been observing in his backyard. Similar to Jacques d'Ortous de Mairan over two centuries 
The Life and Work of Jeffrey C. Hall, Michael Rosbash, and Michael W. Young

before him, Young had been observing cyclical changes of a plant. The plant Young was observing opened its flowers at night and closed them during the day (Young 2009). With the help of the book from his parents, Young was able to learn the why behind the plants' movements, but nothing in the book explained the how. Since then Young has been fascinated by biological clocks (Gruber Foundation 2009b).

While in high school, Young's family move to Dallas, TX. He stayed in Texas to attend college, going to the University of Texas at Austin where he earned his undergraduate degree in biology in 1971 and his doctorate degree in genetics in 1975 . Young then studied transposable elements during his postdoctoral fellowship at Stanford University in Palo Alto, CA from 1975 to 1977 (Gruber Foundation 2009b). In 1978, Young became an Andre and Bella Meyer Foundation fellow and an assistant professor at Rockefeller University in New York City, New York. Young has stayed at Rockefeller ever since. He was also a Howard Hughes Medical Institute Investigator from 1987 to 1997. He is currently a Richard and Jeanne Fisher Professor, Vice President for Academic Affairs, and head of laboratory of genetics at Rockefeller University. Rockefeller has a long history of Nobel winners, of which Young is the 25th (Rockefeller University 2017). There are currently five additional Rockefeller faculty members who are also Nobel Laureates: Roderick MacKinnon (Chemistry 2003), Paul Nurse (Physiology or Medicine 2001), Paul Greengard (Physiology or Medicine 2000), Günter Blobel (Physiology or Medicine 1999), and Torsten Wiesel (Physiology or Medicine 1981) (Nobelprize.org).

During the Rockefeller press conference Young was described as kind, humble, and committed to science. He was introduced with the line "I don't know if the Nobel Prize has ever been given to a nicer person" (Rockefeller University 2017). During his interview with nobelprize.org this kindness and humility came out as he stated multiple times that he was very lucky. He thanked Seymour Benzer and Ron Konopka whom he worked under, as well as his grad students and postdocs. He also thanked the "tremendous mutants," the flies (Young 2017). He thanked all of these people again including the 
The Life and Work of Jeffrey C. Hall, Michael Rosbash, and Michael W. Young

community at Rockefeller University during the Rockefeller press conference, adding that everyone is to be recognized. During the press conference he was asked about the practical pay offs of his work. Young responded that it provides scientists a way of thinking they did not have before, and a target to focus on (Rockefeller University 2017).

As previously noted, Young shares four additional awards besides the Nobel with Hall and Rosbash: the Gruber Prize in Neuroscience (2009), the Louisa Gross Horwitz Prize for Biology or Biochemistry (2011), Shaw Prize in Life Science and Medicine (2013), and the Wiley Prize in Biomedical Sciences (2013). Beyond these, he has numerous other awards and honors. He is a member of the National Academy of Sciences and fellow of the American Academy of Microbiology (Rockefeller University 2017). Some of his awards include the Pittendrigh/Aschoff Award from the Society for Research on Biological Rhythms (2006), the National Institutes of Health MERIT Award (2007), the Canada Gairdner International Award (2012), and the Massry Prize (2012) (Gruber Foundation 2009b, Rockefeller Univesrity 2017). While Young continues his work at Rockefeller, his wife Laurel works down the street as a professor of Biology at Hunter College (Hunter College, Shaw Prize Foundation 2013b). They have two daughters, Natalie and Arissa.

\section{BIBLIOMETRICS}

When it comes to amassing publication and citation information for researchers it is difficult, if not impossible, to get it $100 \%$ correct. Web of Science's organization and search interface make the process easier to ensure the data being collected is accurate. However, it gets complicated for those researchers with more common names. In the case of Michael W. Young, Rockefeller University has two faculty with the same name who have publications indexed in WoS. By eliminating WoS categories such as ethnic studies and linguistics, the author was able to remove most of the publications from the other Michael 
W. Young. In addition, all publications written before 1976 were eliminated since it appears that the MWY in question first published in 1977.

With these constraints in place, Michael W. Young, 2017 Nobel Laureate, has 105 articles indexed in WoS as of November 11, 2017. Beginning in 1977 Young has published almost every year. He has a lower number of publications annually than Hall and Rosbash with his most productive year being 2000 with eight publications (see Figure 7). However, his 105 articles are highly cited with a total of 11,576 citations, averaging 110 citations per publication. These citations have grown over the years reaching a peak in the early 2000's (see Figure 8). His WoS h-index is fifty-three.

[Figure 7 Young Publications]

FIGURE 7 Michael W. Young, Number of Published Items in Each Year. Data source: Web of Science. Retrieved 11 November 2017.

[Figure 8 Young Citations]

FIGURE 8 Michael W. Young, Number of Times Cited in Each Year. Data source: Web of Science. Retrieved 11 November 2017.

The majority of Young's articles have been cited numerous times. His most cited article is his 2001 Time Zones: A Comparative Genetics of Circadian Clocks with 737 citations. His other top cited articles all have well over 300 citations each (see Table 5). Young publishes his articles in a variety of journals, publishing in Neuron eleven times (see Table 6).

Table 5. Top Five Most Highly Cited Papers by Michael W. Young.

[Table 5] 
The Life and Work of Jeffrey C. Hall, Michael Rosbash, and Michael W. Young

Table 6. Top Five Journals Published in by Michael W. Young.

[Table 6]

\section{CONCLUSION}

On October 2, 2017, Jeffrey C. Hall, Michael Rosbash, and Michael W. Young were announced as the awardees of the 2017 Nobel Prize in Physiology or Medicine. These three scientists identified the genes involved in controlling circadian rhythms and discovered how they all work together with oscillating feedback loops. Their work spawned the field of chronobiology and provides current researchers the knowledge and tools necessary to dig deeper in to circadian biology. The biological clock has been shown to be involved in at least half of all gene expression in the human body (Brandeis University 2017c). With a deeper understanding of circadian rhythms, scientists may discover ways to improve sleep, health, and lifestyles. Recent studies have already tied circadian rhythms to diseases including sleep disorders, cancer, and cardiovascular disorders. 
The Life and Work of Jeffrey C. Hall, Michael Rosbash, and Michael W. Young

\section{REFERENCES}

Alibhai, Faisal J., Jonathan LaMarre, Cristine J. Reitz, Elena V. Tsimakouridze, Jeffrey T. Kroetsch, SteffenSebastian Bolz, Alex Shulman, Samantha Steinberg, Thomas P. Burris, Gavin Y. Oudit, and Tami A. Martino. 2017. Disrupting the key circadian regulator clock leads to age-dependent cardiovascular disease. Journal of Molecular and Cellular Cardiology 105:24-37. doi: 10.1016/j.yjmcc.2017.01.008.

Allada, Ravi, Neal E. White, W. Venus So, Jeffrey C. Hall, and Michael Rosbash. 1998. A mutant Drosophila homolog of mammalian clock disrupts circadian rhythms and transcription of period and timeless. Cell 93 (5):791-804. doi: 10.1016/s0092-8674(00)81440-3.

Bargiello, Thaddeus A., F. Rob Jackson, and Michael W. Young. 1984. Restoration of circadian behavioural rhythms by gene transfer in Drosophila. Nature 312 (5996):752-754.

Benzer, Seymour. 1967. Behavioral mutants of Drosophila isolated by countercurrent distribution. Proceedings of the National Academy of Sciences of the United States of America 58 (3):1112-\&. doi: 10.1073/pnas.58.3.1112.

Brandeis University. Jeff Hall professor emeritus of biology. Brandeis University, accessed November 16. http://www.brandeis.edu/facultyguide/person.html?emplid=c383163d56224db4889d61b6eb98 $\underline{\operatorname{acb} 133 f c b 233}$.

---. Michael Rosbash. Brandeis University, accessed November 16.

http://www.brandeis.edu/facultyguide/person.html?emplid=8b3d145edfd753f5a3b4510ab305 3ddd6eeffdbc.

---. 2017a. Jeffrey C. Hall, Ph.D. Brandeis University, accessed October 20. http://www.bio.brandeis.edu/faculty/hall.html.

---. 2017b. Michael Rosbash, Ph.D. Brandeis University, accessed October 20. http://www.bio.brandeis.edu/faculty/rosbash.html. 
The Life and Work of Jeffrey C. Hall, Michael Rosbash, and Michael W. Young

---. 2017c. Press conference with Nobel Prize recipients Michael Rosbash, the Peter Gruber endowed chair in neuroscience, and professor emeritus of biology Jeffrey C. Hall: Brandeis University. Cajochen, Christian, Sylvia Frey, Doreen Anders, Jakub Späti, Matthias Bues, Achim Pross, Ralph Mager, Anna Wirz-Justice, and Oliver Stefani. 2011. Evening exposure to a light-emitting diodes (LED)backlit computer screen affects circadian physiology and cognitive performance. Journal of Applied Physiology 110 (5):1432-1438. doi: 10.1152/japplphysiol.00165.2011.

Columbia University. The Louisa Gross Horwitz prize for biology or biochemistry. Columbia University Medical Center, accessed November 16. http://www.cumc.columbia.edu/research/horwitzprize/prize-awardees.

Cooley, Lynn, Richard Kelley, and Allan Spradling. 1988. Insertional mutagenesis of the Drosophila genome with single P elements. Science 239 (4844):1121-1128.

Dushay, Mitchell S., Michael Rosbash, and Jeffrey C. Hall. 1989. The disconnected visual-system mutations in Drosophila-melanogaster drastically disrupt circadian-rhythms. Journal of Biological Rhythms 4 (1):1-27. doi: 10.1177/074873048900400101.

Edery, Isaac, Laurence J Zwiebel, Marie E Dembinska, and Michael Rosbash. 1994. Temporal phosphorylation of the Drosophila period protein. Proceedings of the National Academy of Sciences 91 (6):2260-2264.

Emery, Patrick, W. Venus So, Maki Kaneko, Jeffrey C. Hall, and Michael Rosbash. 1998. CRY, a Drosophila clock and light-regulated cryptochrome, is a major contributor to circadian rhythm resetting and photosensitivity. Cell 95 (5):669-679. doi: 10.1016/s0092-8674(00)81637-2.

Froy, Oren. 2017. Circadian rhythms, nutrition and implications for longevity in urban environments. Proceedings of the Nutrition Society:1-7. 
The Life and Work of Jeffrey C. Hall, Michael Rosbash, and Michael W. Young

Gekakis, Nicholas, Lino Saez, Anne-Marie Delahaye-Brown, Michael P. Myers, Amita Sehgal, Michael W. Young, and Charles J. Weitz. 1995. Isolation of timeless by per protein interaction: Defective interaction between timeless protein and long-period mutant per ${ }^{\mathrm{L}}$. Science 270 (5237):811.

Green, Amit, Merav Cohen-Zion, Abraham Haim, and Yaron Dagan. 2017. Evening light exposure to computer screens disrupts human sleep, biological rhythms, and attention abilities.

Chronobiology International 37 (7):855-865. doi: 10.1080/07420528.2017.1324878.

Gruber Foundation. 2009a. Michael Rosbash. The Gruber Foundation, accessed November 16. http://gruber.yale.edu/neuroscience/michael-rosbash.

---. 2009b. Michael W. Young. Gruber Foundation, accessed November 17. http://gruber.yale.edu/neuroscience/michael-w-young.

Hall, Jeffrey C. 2015. Ronald J. Konopka in memoriam. Journal of Biological Rhythms 30 (2):71-75. doi: $10.1177 / 0748730415579136$.

---. 2017. Telephone interview with Jeffrey C. Hall. edited by Adam Smith: Nobel Media AB.

Hardin, Paul E, Jeffrey C Hall, and Michael Rosbash. 1990. Feedback of the Drosophila period gene product on circadian cycling of its messenger RNA levels. Nature 343 (6258):536-540.

Howard Hughes Medical Institute. Clockwork genes: Discoveries in biological time. Howard Hughes Medical Institute, accessed November 19. http://www.hhmi.org/biointeractive/clockworkgenes-discoveries-biological-time.

Hu, Youna, Alena Shmygelska, David Tran, Nicholas Eriksson, Joyce Y. Tung, and David A. Hinds. 2016. GWAS of 89,283 individuals identifies genetic variants associated with self-reporting of being a morning person. 7:10448. doi: 10.1038/ncomms10448.

Hunter College. Department of biological sciences: Hunter college. Hunter College, accessed November 17. 
The Life and Work of Jeffrey C. Hall, Michael Rosbash, and Michael W. Young

$\underline{\text { http://biology.hunter.cuny.edu/index.php?option }=\text { com } \text { content } \& \text { view=article } \& i d=55 \& \mid t e m i d=4}$

4.

Ibáñez, Carlos. 2017. The 2017 Nobel prize in physiology or medicine - advanced information:

Discoveries of molecular mechanisms controlling the circadian rhythm In Nobelprize.org.

Irish, Leah A., Christopher E. Kline, Heather E. Gunn, Daniel J. Buysse, and Martica H. Hall. 2015. The role of sleep hygiene in promoting public health: A review of empirical evidence. Sleep medicine reviews 22:23-36. doi: 10.1016/j.smrv.2014.10.001.

Khalyfa, Abdelnaby, Valeriy A. Poroyko, Valeriy H. Qiao, Aex Gileles-Hillel, Ahamed A. Khalyfa, Mahzad Akbarpour, Isaac Almendros, Ramon Farre, and David Gozal. 2017. Exosomes and metabolic functionin mice exposed to alternating dark-light cycles mimicking night shift work schedules. Frontiers in Physiology 8. doi: 10.3389/fphys.2017.00882.

Kloss, Brian, Jeffrey L. Price, Lino Saez, Justin Blau, Adrian Rothenfluh, Cedric S. Wesley, and Michael W Young. 1998. The Drosophila clock gene double-time encodes a protein closely related to human casein kinase I epsilon. Cell 94 (1):97-107. doi: 10.1016/s0092-8674(00)81225-8.

Kolata, Gina. 2017. 2017 Nobel prize in medicine goes to 3 Americans for body clock studies. The New York Times. Accessed October 9, 2017.

Konopka, Ronald J. 1986. Neurogenetics of Drosophila circadian rhythms. In Evolutionary genetics of invertebrate behavior, 215-221. Springer.

Konopka, Ronald J., and Seymour Benzer. 1971. Clock mutants of Drosophila -melanogaster. Proceedings of the National Academy of Sciences of the United States of America 68 (9):2112-\&. doi: $10.1073 /$ pnas.68.9.2112.

Konopka, Ronald J., Steven Wells, and Thomas Lee. 1983. Mosaic analysis of a Drosophila clock mutant. Molecular and General Genetics MGG 190 (2):284-288. 
The Life and Work of Jeffrey C. Hall, Michael Rosbash, and Michael W. Young

Kyriacou, Charalambos P., and Jeffrey C Hall. 1980. Circadian-rhythm mutations in Drosophila melanogaster affect short-term fluctuations in the males courtship song. Proceedings of the National Academy of Sciences of the United States of America-Biological Sciences 77 (11):67296733. doi: 10.1073/pnas.77.11.6729.

Li, Guolin, Cen Xie, Siyu Lu, Robert G. Nichols, Yuan Tian, Licen Li, Daxeshkumar Patel, Yinyan Ma, Chad N. Brocker, and Tingting Yan. 2017. Intermittent fasting promotes white adipose browning and decreases obesity by shaping the gut microbiota. Cell metabolism 26 (4):672-685. e4.

Mahmood, Tahrin, and Ping-Chang Yang. 2012. Western blot: Technique, theory, and trouble shooting. North American Journal of Medical Sciences 4 (9):429-434. doi: 10.4103/1947-2714.100998.

Manning, Aubrey. 1965. Drosophila and the evolution of behaviour. In Viewpoints in biology, edited by J.D. Carthy and C.L Duddington, 125-169. London: Butterworth \& Co. LTD.

Montaigne, David, Xavier Marechal, Thomas Modine, Augustin Coisne, Stéphanie Mouton, Georges Fayad, Sandro Ninni, Cédric Klein, Staniel Ortmans, Claire Seunes, Charlotte Potelle, Alexandre Berthier, Celine Gheeraert, Catherine Piveteau, Rebecca Deprez, Jérome Eeckhoute, Hélène Duez, Dominique Lacroix, Benoit Deprez, Bruno Jegou, Mohamed Koussa, Jean-Louis Edme, Philippe Lefebvre, and Bart Staels. 2017. Daytime variation of perioperative myocardial injury in cardiac surgery and its prevention by Rev-Erb $\alpha$ antagonism: A single-centre propensity-matched cohort study and a randomised study. The Lancet. doi: 10.1016/S0140-6736(17)32132-3.

Morgan, Thomas H. 1910. Sex limited inheritance in Drosophila. Science 32 (1):120-122. doi: 10.1126/science.32.812.120.

Myers, Michael P., Lino Saez, Adrian Rothenfluh-Hilfiker, and Michael W. Young. 1997. The molecular basis for circadian timekeeping in Drosophila. Journal of Neurochemistry 69:S118-S118. 
The Life and Work of Jeffrey C. Hall, Michael Rosbash, and Michael W. Young

Myers, Michael P., Karen Wager-Smith, Adrian Rothenfluh-Hilfiker, and Michael W Young. 1996. Lightinduced degradation of timeless and entrainment of the Drosophila circadian clock. Science 271 (5256):1736-1740. doi: 10.1126/science.271.5256.1736.

Nobelprize.org. Hermann j. Muller - facts. Nobel Media AB 2014, accessed October 23. https://www.nobelprize.org/nobel prizes/medicine/laureates/1946/muller-facts.html.

---. Nobel laureates and country of birth. Nobel Media AB 2014, accessed October 24. https://www.nobelprize.org/nobel prizes/lists/countries.html.

---. The Nobel prize in physiology or medicine 1933. Nobel Media AB 2014, accessed October 23. https://www.nobelprize.org/nobel prizes/medicine/laureates/1933/.

---. 1995. Physiology or medicine 1995 - press release. Nobel Media AB 2014, accessed October 23. https://www.nobelprize.org/nobel prizes/medicine/laureates/1995/press.html.

---. 2011. The 2011 Nobel prize in physiology or medicine - press release. Nobel Media AB 2014, accessed October 23. https://www.nobelprize.org/nobel prizes/medicine/laureates/2011/press.html.

---. 2017a. The 2017 Nobel prize in physiology or medicine - press release. Nobel Media AB Last Modified 10/02/2017, accessed October 09. https://www.nobelprize.org/nobel prizes/medicine/laureates/2017/press.html.

---. 2017b. Announcement of the 2017 Nobel prize in physiology or medicine. Nobel Media AB accessed October 09. https://www.nobelprize.org/mediaplayer/index.php?id=2720.

Nuzzo, Regina. 2005. Profile of Jeffrey C. Hall. Proceedings of the National Academy of Sciences of the United States of America 102 (46):16547-16549. doi: 10.1073/pnas.0508533102.

Patke, Alina, Patricia J Murphy, Onur Emre Onat, Ana C Krieger, Tayfun Özçelik, Scott S Campbell, and Michael W Young. 2017. Mutation of the human circadian clock gene cry1 in familial delayed sleep phase disorder. Cell 169 (2):203-215. e13. 
The Life and Work of Jeffrey C. Hall, Michael Rosbash, and Michael W. Young

Plautz, Jeffrey D., Maki Kaneko, Jeffrey C. Hall, and Steve A. Kay. 1997. Independent photoreceptive circadian clocks throughout Drosophila. Science 278 (5343):1632.

Pray, Leslie A. 2008. Transposons: The jumping genes. Nature Education 1 (1):204.

Price, Jeffrey L., Justin Blau, Adrian Rothenfluh, Marla Abodeely, Brian Kloss, and Michael W. Young. 1998. Double-time is a novel Drosophila clock gene that regulates period protein accumulation. Cell $94(1): 83-95$.

Rockefeller University. 2017. Rockefeller university biologist Michael W. Young honored with Nobel prize for pioneering studies on circadian rhythm. The Rockefeller University, accessed October 20. https://www.rockefeller.edu/news/20468-rockefeller-university-biologist-michael-w-younghonored-nobel-prize-discovery-molecular-mechanisms-controlling-circadian-rhythm/.

Rockefeller Univesrity. 2017. Michael W. Young, Ph.D. The Rockefeller University, accessed October 20. https://www.rockefeller.edu/our-scientists/heads-of-laboratories/914-michael-w-young/.

Roenneberg, Till, and Martha Merrow. 2016. The circadian clock and human health. Current Biology 26 (10):R432-R443. doi: https://doi.org/10.1016/i.cub.2016.04.011.

Rosbash, Michael. 2017. Telephone interview with Michael Rosbash. edited by Adam Smith: Nobel Media AB.

Rothenfluh, Adrian, Marla Abodeely, and Michael W. Young. 2000. Short-period mutations of per affect a double-time-dependent step in the Drosophila circadian clock. Current Biology 10 (21):13991402. doi: 10.1016/s0960-9822(00)00786-7.

Rutila, Joan E., Vipin Suri, Myai Le, W. Venus So, Michael Rosbash, and Jeffrey C. Hall. 1998. CYCLE is a second bHLH-PAS clock protein essential for circadian rhythmicity and transcription of Drosophila period and timeless. Cell 93 (5):805-814. doi: 10.1016/s0092-8674(00)81441-5.

Schernhammer, Eva S., Francine Laden, Frank E. Speizer, Walter C. Willett, David J. Hunter, Ichiro Kawachi, and Graham A. Colditz. 2001. Rotating night shifts and risk of breast cancer in women 
The Life and Work of Jeffrey C. Hall, Michael Rosbash, and Michael W. Young

participating in the nurses' health study. Journal of the National Cancer Institute 93 (20):15631568.

Sehgal, Amita, Bernice Man, Jeffrey L. Price, Leslie B. Vosshall, and Michael W. Young. 1991. New clock mutations in Drosophila. Annals of the New York Academy of Sciences 618:1-10. doi: 10.1111/j.1749-6632.1991.tb27233.x.

Sehgal, Amita, Jeffrey L. Price, Bernice Man, and Michael W. Young. 1994. Loss of circadian behavioral rhythms and per RNA oscillations in the Drosophila mutant timeless. Science 263 (5153):1603. doi: 10.1126/science.8128246.

Sehgal, Amita, Jeffrey L. Price, and Michael W. Young. 1992. Ontogeny of a biological clock in Drosophila -melanogaster. Proceedings of the National Academy of Sciences of the United States of America 89 (4):1423-1427. doi: 10.1073/pnas.89.4.1423.

Shang, Yuhua, Paula Haynes, Nicolas Pirez, Kyle I. Harrington, Fang Guo, Jordan Pollack, Pengyu Hong, Leslie C. Griffith, and Michael Rosbash. 2011. Imaging analysis of clock neurons reveals light buffers the wake-promoting effect of dopamine. Nat Neurosci 14 (7):889-895. doi: http://www.nature.com/neuro/journal/v14/n7/abs/nn.2860.html\#supplementary-information.

Shaw Prize Foundation. 2013a. Autobiography of Michael Rosbash. The Shaw Prize Foundation, accessed November 16.

http://www.shawprize.org/en/shaw.php?tmp=3\&twoid=94\&threeid=219\&fourid=386\&fiveid=1

83.

---. 2013b. Autobiography of Michael Young. The Shaw Prize Foundation, accessed November 16. http://www.shawprize.org/en/shaw.php?tmp=3\&twoid=94\&threeid=219\&fourid=386\&fiveid=1 $\underline{84}$. 
The Life and Work of Jeffrey C. Hall, Michael Rosbash, and Michael W. Young

---. 2013c. Life science and medicine announcement and citation. The Shaw Prize Foundation, accessed November 16.

http://www.shawprize.org/en/shaw.php?tmp=3\&twoid=94\&threeid=219\&fourid=369.

Siwicki, Kathleen K, Cheryl Eastman, Gabriele Petersen, Michael Rosbash, and Jeffrey C Hall. 1988. Antibodies to the period gene product of Drosophila reveal diverse tissue distribution and rhythmic changes in the visual system. Neuron 1 (2):141-150.

Stephenson, Rob, and Neil H Metcalfe. 2013. Drosophila melanogaster: A fly through its history and current use. The journal of the Royal College of Physicians of Edinburgh 43 (1):70-75.

Sturtevant, AH. 1915. Experiments on sex recognition and the problem of sexual selection in Drosophila. Journal of Animal Behavior 5 (5):351.

Tomoda, Akemi, Teruhisa Miike, Keiko Uezono, and Terukazu Kawasaki. 1994. A school refusal case with biological rhythm disturbance and melatonin therapy. Brain \& Development 16 (1):71-76. doi: 10.1016/0387-7604(94)90117-1.

University of Maine. 2017. Jeff Hall receives Nobel prize in physiology or medicine. The University of Maine, accessed October 24. https://sbe.umaine.edu/2017/10/02/jeff-hall-receives-nobel-prizephysiology-medicine/.

Vosshall, Leslie B., Jeffrey L. Price, Amita Sehgal, Lino Saez, and Michael W. Young. 1994. Block in nuclear localization of period protein by a second clock mutation, timeless. (circadian clock)(cover story). Science 263 (5153):1606-1610.

Walch, Olivia J., Amy Cochran, and Daniel B. Forger. 2016. A global quantification of "normal" sleep schedules using smartphone data. Science Advances 2 (5).

Ward, Ritchie R. 1971. The living clocks. 1 ed. New York, NY: Alfred A. Knopf, Inc.

Wedell, Anna 2017. Interview about the 2017 Nobel prize in physiology or medicine. edited by Lotta Fredholm: Nobel Media AB. 
The Life and Work of Jeffrey C. Hall, Michael Rosbash, and Michael W. Young

Wiley. 2013. Twelfth annual Wiley prize in biomedical sciences awarded to Dr. Michael Young, Dr. Jeffrey Hall and Dr. Michael Rosbash. Wiley, accessed November 16. http://www.wiley.com/WileyCDA/PressRelease/pressReleaseld-107171.html.

Yeh, Jennifer. 2002. Circadian rhythm. In Animal sciences, edited by Allan B. Cobb, 149-151. New York: Macmillan Reference USA.

Young, Michael W. 2009. Michael Young. Current Biology 19 (9):R352-R353. doi: https://doi.org/10.1016/j.cub.2009.03.039.

---. 2017. Telephone interview with Michael W. Young. edited by Adam Smith: Nobel Media AB.

Young, Michael W., and Steve A. Kay. 2001. Time zones: A comparative genetics of circadian clocks. Nature Reviews Genetics 2 (9):702-715.

Zehring, William A., David A. Wheeler, Pranhitha Reddy, Ronald J. Konopka, Charalambos P. Kyriacou, Michael Rosbash, and Jeffrey C. Hall. 1984. P-element transformation with period locus DNA restores rhythmicity to mutant, arrhythmic Drosophila melanogaster. Cell 39 (2):369-376.

Zeng, Hongkui, Zuwei Qian, Michael P. Myers, and Michael Rosbash. 1996. A light-entrainment mechanism for the Drosophila circadian clock. Nature 380 (6570):129-135. 